\title{
MEASURES UNDERTAKEN FOR THE MANAGEMENT OF OBSTRUCTIVE UROPATHY IN ADVANCED CARCINOMA CERVIX
}

\author{
MASUD AHMED ${ }^{1}$, MAAWAL $^{2}$, MA RASHID 2 , MD. SHAHADAT HOSSAIN ${ }^{2}$, S RAHMAN $^{3}$, \\ MD. NURUL HOODA ${ }^{4}$, ATM AMAN ULLAH ${ }^{5}$, MD. SAYEDUL ISLAM ${ }^{5}$ \\ ${ }^{1}$ National Institute of Cancer Research \& Hospital, Mohaklhali, Dhaka, ${ }^{2}$ Dhaka Medical College and Hospital, Dhaka, \\ ${ }^{3}$ BIRDEM Hospital, Dhaka, ${ }^{4}$ Department Urology, NIKDU, Dhaka, ${ }^{5}$ Department of Urology, BSMMU, Dhaka
}

\begin{abstract}
Objectives: To find out the procedures adopted for the management of obstructive Uropathy in advanced Carcinoma cervix and To identify the most excellent option for their management.

Methods: It was a retrospective cross sectional study. Data were collected from patient's record of Urology outpatient department (OPD) and indoor of Dhaka Medical College Hospital (DMCH) for the year 2010. Patients attended with other causes of obstructive uropathy were excluded.

Results: Total 32 patients were included in this study. Mean age of the patients was $46 \pm 6.90$ years. All of them were squamous cell carcinoma of stage III \& IV disease. About $60 \%$ patients received pre-operative radiotherapy for cervical cancer. Twenty three patients underwent retrograde ureteral stenting, 6 patients underwent percutaneous nephrostomy $(P C N)$ and rest 3 underwent cutaneous ureterostomy. Mean pre-operative serum creatinin was $2.68 \pm .24 \mathrm{mg} / \mathrm{dl}$. and post-operatively it dropped to $1.30 \pm .05 \mathrm{mg} / \mathrm{dl}$. which is statistically very much significant. Post-operative hydronephrosis also improved significantly following operations. Difference observed in the improvement of renal function or hydronephrosis among the surgical procedures but it was not statistically significant.

Conclusion: Any of the surgical procedures undertaken for the management of obstructive uropathy due to advanced carcinoma of cervix, provided significant improvement of renal function and hydronephrosis.
\end{abstract}

Key word: Obstructive uropathy; Carcinoma cervix.

Bangladesh J. Urol. 2012; 15(1): 15-17

\section{Introduction}

Cancer of uterine cervix is one of the leading causes of cancer death among women worldwide. The estimated new cancer cases per year are $5,00,000$ of which $79 \%$ occurs in developing countries. In urban areas of India, cancer of cervix accounts for over $40 \%$, of which $65 \%$ accounts in rural areas. About $70 \%$ of them present as locally advanced disease \& one third of them with renal failure ${ }^{1}$. Based on the data available from the Radiotherapy Department of Dhaka Medical College Hospital, relative percentage of cervical cancer is 24 per 1, 00,000 populations. It is the most common cancer affecting women of Bangladesh ${ }^{2}$. Most common cause of death in advanced Carcinoma cervix is uraemia due to obstructive uropathy ${ }^{3}$.

Correspondence: Dr. Masud Ahmed, Department of Urology, National Institute of Cancer Research \& Hospital, Mohaklhali, Dhaka. E-mail: uromasud@yahoo.com
This is due to either external compression or malignant involvement of lower ureter ${ }^{4}$. Ureteral obstruction due to malignancy carries a poor prognosis with resulting median survival of three to seven months. So, some palliative urinary diversion procedures are essential part of management of these patients ${ }^{1}$.If the obstruction in the urinary tract is not removed, patient's clinical condition will deteriorate at a fast pace through uraemia, water-electrolyte abnormalities \& urinary infection with a consequent reduction of alertness and subsequent death ${ }^{5}$. Currently, retrograde ureteral clearing with double-J ureteral stent is the most widely used technique for relieving obstruction of urinary tract. However, the retrograde ureteral stenting is sometimes impossible in cancer patients due to the presence of anatomic abnormalities, bleeding or ureteral compression ${ }^{5}$. Stenting is very simple and easy procedure. Usually, it is performed with spinal anaesthia, but can be performed 
with local anaesthesia also. There is no need of external appliance for urine collection. But as it is an endoscopic procedure, so expensive endoscopic instruments are necessary for the operative procedure.

Percutaneous nephrostomy (PCN) is also an easy procedure. It can be performed by local anaesthesia but not free of complications. About 31\% patients' presents with loss of nephrostomy catheter \& $19 \%$ shows urinary infection ${ }^{5}$. The procedure is performed under ultrasound or CT guidance. When pelvi-calyceal dilatation is very minimum, $\mathrm{PCN}$ is a bit difficult. Occasionally, some open surgical procedures like Cutaneous ureterostomy or Ureteroneocystostomy have to accept. These open procedures have to perform under spinal or general anaesthia. But sometimes patients are not fit for anaesthesia. The present study aims to observe any difference in the effectiveness of various management procedures adopted for advanced carcinoma Cervix.

\section{Methods}

It was a retrospective Cross sectional study. Data were collected from hospital record in a structured questioner. Patients recorded at Urology OPD and indoor of Dhaka Medical College Hospital (DMCH) with diagnosis of obstructive uropathy due to advanced carcinoma cervix within the period of $1^{\text {st }}$ January 2010 to 31 st December 2010 -were included in this study. Documents of patients with other causes of obstructive uropathy were excluded. All records that meet the selection criteria were included. Following collection of data it was analysed by computer software Statistical Package for the Social Sciences (SPSS version 16).

\section{Results}

Total 32 patients were included in this study. Age distribution of patients shown in the following histogram. Mean age was $46 \pm 6.90$ years.

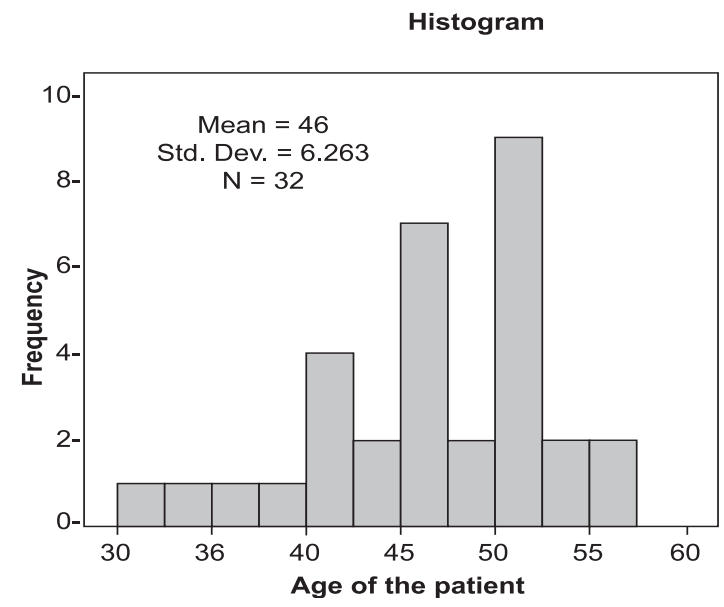

Fig.-1: Age distribution of the patients.
All of them were squamous cell carcinoma type. About $50 \%$ (16) were stage III and rest were stage IV disease. Out of 32 patients 19(59.38\%) patients received radiotherapy pre-operatively. About $72 \%(23)$ patients underwent ureteral stenting, six patients underwent PCN and rest 3 patients underwent cutaneous ureterostomy. Post-operative hydronephrosis improved significantly $(P<.001)$ in comparison with pre-operative hydronephrosis.

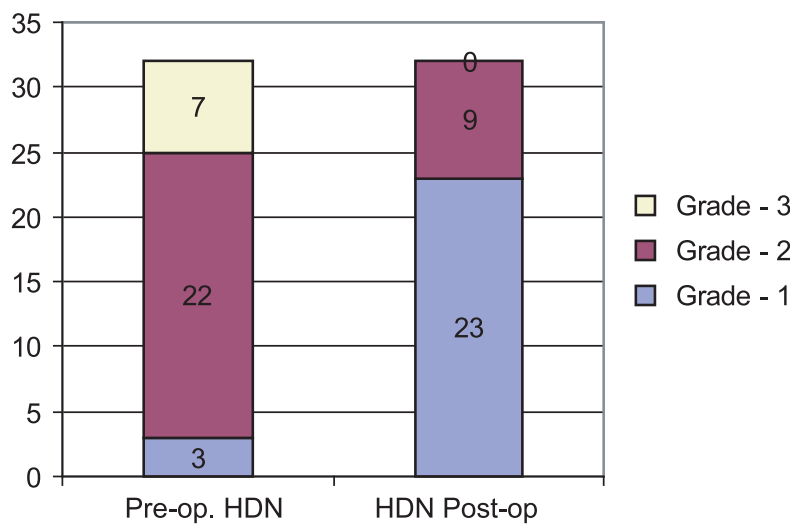

Fig.-2: Pre and post-operative grade of hydronephrosis.

Mean pre-operative serum creatinin was $2.68 \pm .24 \mathrm{mg} /$ $\mathrm{dl}$. and post-operative $1.30 \pm .05 \mathrm{mg} / \mathrm{dl}$.-which is statistically very significant $(P<.001)$.

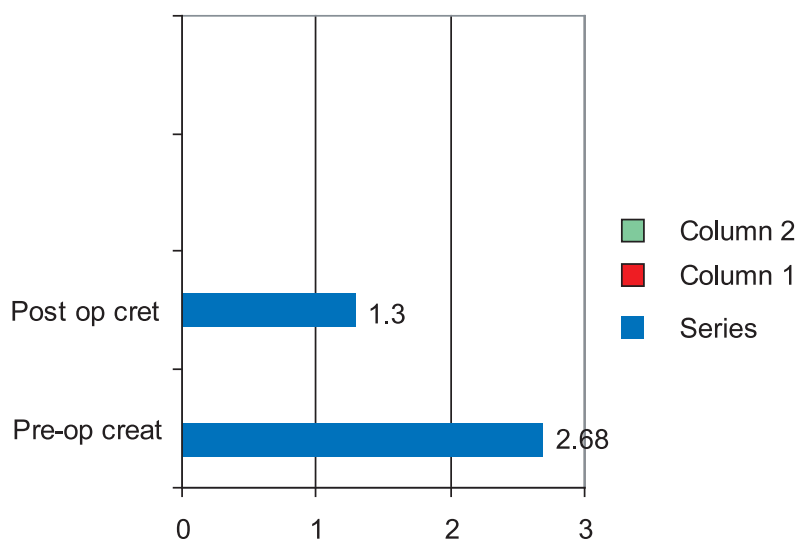

Fig.-3: Pre and post-operative creatinin level.

Post-operative hydronephrosis improved significantly in comparison with pre-operative level of hydronephrosis. 


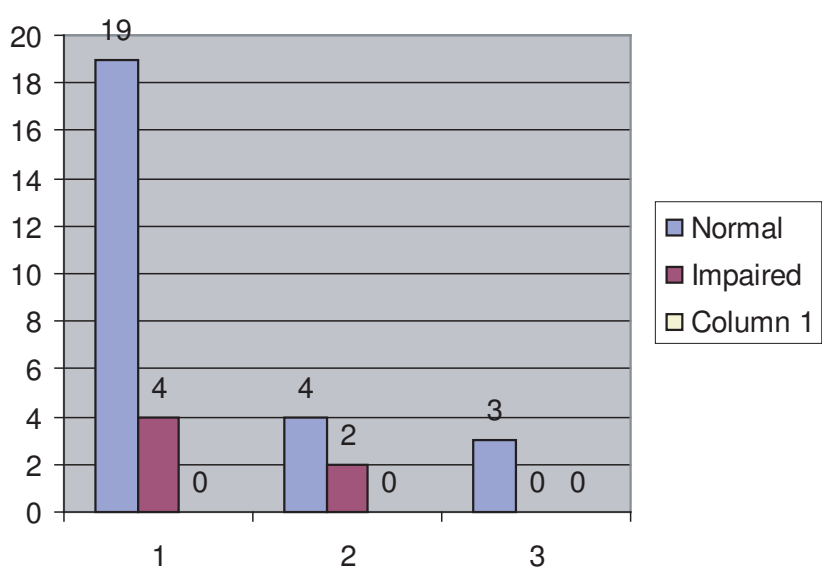

Fig.-4: Improvement of renal function following surgical procedures.

\section{Discussion}

According to Cancer Registry Report, National Institute of Cancer Research and Hospital (NICRH) 2005-2007 average age of cancer cervix was 46 years $^{6}$. The present study showed exact similar result of mean age 46 years.

In that report of $\mathrm{NICRH}$, tumour Board offered radiotherapy to $75 \%$ of patients attended with cervical cancer of different stage ${ }^{6}$. Similarly, this study showed about $60 \%$ patients received radiotherapy for advance staged carcinoma cervix before surgical procedures. At postoperative period serum creatinin dropped to normal at majority of the patients. Janaki and collegue ${ }^{1}$ reported that serum creatinin dropped to normal within one month of percutaneous nephrostomy. Keidan $R D^{8}$ showed $85 \%$ improvement of renal function following percutaneous nephrostomy. Present study showed that creatinin dropped from $2.68 \mathrm{mg} / \mathrm{dl}$ to $1.30 \mathrm{mg} / \mathrm{dl}$. which is very much similar to other studies. Then they offered radiotherapy and chemotherapy as per schedule.

$\mathrm{NICRH}$, Dhaka reported that about $90 \%$ of cervical cancers were squamous cell carcinoma ${ }^{6}$. Current study report showed all patients attended with obstructive uropathy due to carcinoma cervix are squamous cell carcinoma.

\section{Conclusion}

Any of the described surgical procedures adopted for the management of obstructive uropathy due to advanced carcinoma cervix may provide the patients very good result in terms of improving renal function and hydronephrosis.

\section{Recommendations}

This study recommended further study at large scale with adequate time and fund. The knowledge could be disseminated throughout the country among the surgeons, urologists and gynaecologists for better management of obstructive uropathy patients due to advanced carcinoma cervix.

\section{Acknowledgement}

I express my profound gratitude and deep respect to Director, Bangladesh Medical Research Council (BMRC), Dhaka for allowing me to participate in the training program. I am also grateful to Prof. S.M. Mahbubul Alam, Head of the Department of Urology, Dhaka Medical College Hospital, Dhaka, for his kind cooperation and valuable suggestions. I am very much grateful to Prof. Dr. Mollah Obayedullah Baki, Director and professor of radiation oncology for his valuable suggestions and advice in carrying out this work.

\section{Conflict of Interest : None declared}

\section{References}

1. Janaki MG. Mukesh S. Arul Ponni TR. Nirmala S. Aggressive Approach in a Case of Cancer Cervix with Uremia. Indian J Palliat Care. 2010; Jan-Apr; 16(1): 52-53.

2. Banglapedia, National encyclopaedia of Bangladesh, 2006.

3. PPT of Dr Shabnam Naz. (www.authorstream.com)

4. Role of Percutaneous Nephrostomy in Advanced Cervical Carcinoma with Obstructive Uropathy: A Case Series. Mishra K. Desai A. Patel S. Mankad M. and Dave K. Indian J Palliat Care. 2009; Jan-Jun; 15(1): 37-40.

5. Frederico R. Romero; Marcos Broglio; Silvio R. Pires; Roberto F. Roca; Ione A. Guibu; Marjo D. Perez. Indications for percutaneous nephrostomy in patients with obstructive uropathy due to malignant urogenital neoplasias, Int. braz j urol. 2005; Mar./Apr; 31(2)

6. Cancer Registry Report, National Institute of Cancer Research and Hospital 2005-2007.

7. Keidan RD, Greenberg RE, Hoffman JP, Weese $\mathrm{JL}$. Is percutaneous nephrostomy for hydronephrosis appropriate in patients with advanced cancer? .Am J Surg. 1988 Sep;156(3 Pt 1):206-8. 